\section{Arteriovenous malformations}

\section{DONNA WINDERBANK-SCOTT}

St Mary's Hospital, Paddington, London, UK

E: donna@gambyte.co.uk

\section{AVM Support UK}

\section{www.avmsupport.org.uk}

This site is designed as a patient information portal as well as a support forum and concentrates almost exclusively on cerebrovascular AVMs. The site is fairly basic but comprehensive, and the author and date of the last update are recorded on each page. The section on investigations and what to expect is particularly useful for an anxious patient; there are many personal experiences and testimonies to read. A facility for altering text size is useful for the visually impaired and the overall layout is logical and well organised. However, the site is mainly text-based, has few illustrations or pictures and is probably too basic for medical professionals. **

\section{Birthmarks}

\section{www.birthmarks.us}

This site has been written by a vascular malformations' specialist and contains many good, high-quality images including a variety of clinical photographs as well as CT scans, angiography images and $3-D$ reconstructions. The clinical information is broken down into the various types of malformation with separate pages for associated syndromes. Links to full-text PDF journal articles by various authors provide more in depth clinical information to a specialist level. There are Power-Point presentations to download, and several case studies. However, some links provide abstracts instead of full-text articles and there is no facility to enlarge the images. This site is very detailed and comprehensive; as such. it will probably be most useful for post-MRCS surgical trainees or when preparing presentations on a specific aspect of the field. ${ }^{* * *}$

\section{Emedicine.com}

\section{http://www.emedicine.com/plastic/topic468.htm}

From the student staple website, this article provides a brief overview of vascular malformations including history, diagnosis, pathophysiology and aetiology. The information is not particularly detailed and is probably more appropriate for medical students. There are only four images which are very poor quality and not enlargeable without a paid subscription fee. However, the 1-2 line summaries of vascular malformation syndromes are useful. **

\section{Vascular Disease Foundation}

\section{http://www.vdf.org/CVM/}

This American organisation's aim is to educate patients in aspects of vascular disease and risk-factor modification. The website covers all aspects of vascular disease with a section on congenital vascular malformations. The information in this section is patient-orientated but entirely text with no images. There are spelling mistakes and the content is difficult to read, with long, confusing prose. Several sections are still under development and are currently empty. For a large, multidisciplinary organisation, this site is disappointing.*

\section{NOVA - National Organisation of Vascular Anomalies http://www.novanews.org/}

Another American organisation website, which focuses on haemangiomas and birthmarks as well as AVM-related syndromes. There are plenty of photographs and further links to various research projects in this field. The clinical overviews are primarily aimed at patients, but are detailed enough for surgical trainees and contain full references. The site is fairly well laid out but has some formatting problems with text sizes and occasional missing characters. However, overall, the clinical information is very well written and comprehensive. ${ }^{* * *}$

\section{Personal digital assistant (PDA)-based drug formularies}

THOMAS HS FYSH

Department of Surgery, Royal Cornwall Hospital, UK

E: thomas.fysh@btinternet.com

The days of frantically searching for a dog-eared copy of the BNF could soon be over. If you own a PDA or smartphone, the chances are that all this information could be available at your fingertips in an instant. The PDA-based formularies are geared-up mainly for the US/Canadian market and this has practical implications when using them in the UK, specifically in the NHS (such as licensed preparations, trademarks and the use of non-SI units).

\section{$B N F$}

The obvious exception is the original BNF which is compiled by the BMA and Royal Pharmaceutical Society of Great Britain, available for Palm OS. It can be obtained on-line from $<$ www.pharmpress.com $>$ and is updated every 6 months but only comes on an SD card which must be ordered. It is not cheap at $£ 99$ per edition (every 6 months) + VAT for NHS users but has the distinct advantage of being the only formulary here which was designed for the UK. A big disappointment is that, despite pledges to make it available to pocket PC (PPC) since 2004, this has yet to happen. We are promised that it is in the pipeline as is a downloadable version. Unfortunately, there are no plans for a Symbian OS version so Nokia smartphone users won't be able to benefit. The software itself is reliable and easy to use, and the information is comprehensive, as you might expect. Its cost and lack of compatibility let it down. ${ }^{* * *}$

\section{Epocrates}

My personal favourite is Epocrates Rx which is available for free in its most basic (but perfectly adequate) form from 
$<w w w . e p o c r a t e s . c o m>$. Although it probably contains fewer drug monographs (i.e. actual substances rather that trade names) than some of its rivals, I have yet to be let down. UK trade names are also given. Frustratingly, most antibiotic doses are given in units and not milligrams, but this is not unusual for the US-based references. The typical adjuncts such as side-effects, cautions and contra-indications are easily accessed and look to be comprehensive. Epocrates auto-updates when connected to the internet (either through PC synchronisation or direct connection). This includes recent drug advice, cautions and studies which the user must acknowledge before proceeding. A fuller version (Epocrates Essentials) includes disease information and diagnostic software for $\$ 149$ per year. Available for both PPC and Palm (but not Symbian), this is a great find.****

\section{Handheldmed}

For Smartphone/Symbian OS fans, there only seems to be one contender. Using the Mobipocket Reader platform, Handheldmed present 'A to Z Drug Facts' available at $<w w w$.handheldmed.com $>$. It claims to contain over 4600 drugs, costs $\$ 65$ and is also available for PPC and Palm. The Mobipocket platform is easy to install and pleasant to use, allowing the user to launch other programs by Handheldmed (e.g. an abridged Merck Index amongst over 200 other medicine-oriented downloads). It also claims FDA approval which may or may not be comforting to the UK consumer. ***

\section{lexi-drugs}

Another comprehensive formulary is lexi-drugs <www.lexi.com $>$. This US-based formulary contains over 1600 drug monographs and 7000 trade names with dosing, interactions, cautions and special alerts. There is access to unlimited updates throughout year. It also contains a dental formulary, and extras such as abbreviations, natural therapies (which may be why the monograph total is so high), a toxicology database, laboratory investigations, patient advice leaflets and much more. It is a chunky file, requiring $61 \mathrm{MB}$ of free space (stretching the limits of most PPC phones unless a memory card is available) and is also expensive at $\$ 215$ for one year. The even chunkier 'lexicomplete' package includes a medical dictionary and medical consultation program for an extra $\$ 60$. If you can't decide, then a free trial is available.**

\section{Skyscape}

PDA giant Skyscape have also joined in the action, providing three convincing formularies for slightly different audiences (and wallets). Their various products can be downloaded from $<w w w . h a n d a n g o . c o m>$. The flagship product is AHFS (American Hospital Formulary Service) Drug Information. At \$79 per year, this appears to be a close equivalent to our own BNF. This product launches from the smARTlink platform (included) which can also be used to provide a handy cross-referencing service (e.g. literature searches). It is comprehensive but is obviously tailored to the US market and consequently loses appeal in the
UK (especially considering the price tag).** Alternatively, Mosby's Medical Drug Reference (also by Skyscape) is available for download at only $\$ 40$. It has fewer drug monographs than its big brother (800 compared to over 1200 ) but this is still plenty and the price tag is more attractive.*** Somewhere in the middle lies the Physician's Drug Handbook (again, by Skyscape at \$50), which contains 1000 monographs. Considering the obvious drawbacks of using a US-based formulary, it is probably not worth paying too much for one, especially when Epocrates does a perfectly good job for free.**

We seem to be lagging somewhat in the UK as far as PDAbased formularies are concerned. This is probably a reflection on prescribing standards, NHS guidelines and variable drug policies throughout the NHS trusts. The BNF would be the obvious winner but is let down a sky-high price tag and no PPC/Symbian compatibility. We wait in hope. Meanwhile, it is worth remembering that a formulary which is not endorsed by your trust should only be used as an aide-memoir, and not a replacement for the trust formulary or the BNF.

\section{Reflex sympathetic dystrophy}

CS MODI

Leicester Royal Infirmary, Leicester, UK

Reflex sympathetic dystrophy (RSD), also known as complex regional pain syndrome (CRPS), is a difficult condition to recognise and manage. It can be devastating for the patient and may result in long periods of impairment, disability and handicap following an injury or surgery. It is important for the clinician to recognise and treat this condition early as this has been shown to improve the recovery rate. The following selected internet sites provide valuable information on this condition for a variety of readers.

\section{Wheeless' Textbook of Orthopedics \\ http://wheelessonline.com/ortho/}

reflex_sympathetic_dystrophy_complex_regional_pain_syndrome

This is an excellent online resource as a starting point for the specialist. It provides a comprehensive, and yet succinct, guide to all aspects of this condition. The most useful feature of this site is up-to-date links to the current evidence for the treatment modalities available to the clinician, which is important when decision making. The website is well designed and easy to navigate. I would recommend it for the specialist although it is limited by small text and few images (although it makes up for this in other topics).****

American Society for Surgery of the Hand

http://www.assh.org/Am/template.cfm

This American website has three main areas in which to navigate. These are split into Patient and Public, Doctors and Hand Care 